Gazi University
Journal of Science
$\mathrm{http} / /$ dergipark.gov.tr/gujs

\title{
On the Exponentiated Weibull Rayleigh Distribution
}

\author{
Mohammed ElGARHY ${ }^{1, *}$, Ibrahim ELBATAL ${ }^{2,3}$ (i) Gholam HAMEDANI $^{4}$ (D) Amal HASSAN $^{2}$ (i) \\ ${ }^{l}$ Valley High Institute for Management Finance and Information Systems, Obour, Qaliubia, Egypt \\ ${ }^{2}$ Faculty of Graduate Studies for Statistical Research, Department of Mathematical Statistics, Cairo University, Giza, Egypt \\ ${ }^{3}$ College of Science, Al Imam Mohammad Ibn Saud, Department of Mathematics and Statistics, Islamic University \\ ${ }^{4}$ Department of Mathematics, Statistics and Computer Science, Marquette University, WI 53201-1881, Milwaukee, USA
}

\section{Highlights}

- We introduce a new four parameter distribution called exponentiated Weibull Rayligh distribution.

- We study some statistical properties of the new model.

- We use the maximum likelihood method to estimate the parameters of the distribution.

- We make simulation results by using Mathematica 9 .

- The new model is very flexible and include some special models.

\section{Article Info}

Received: $25 / 05 / 2017$

Accepted:25/09/2018

\section{Keywords}

Exponentiated Weibullfamily of distributions Maximum likelihood

Moments

Characterizations

\begin{abstract}
A new four-parameter probability model, referred to the exponentiated Weibull Rayleigh (EWR) distribution, is introduced. Essential statistical properties of the distribution are considered. The maximum likelihood estimators of population parameters are given in case of complete sample. Simulation study is carried out to estimate the model parameters of EWR distribution. Additionally, parameter estimators are given in case of Type II censored samples. We come up with two applications to confirm the usefulness of the proposed distribution.
\end{abstract}

\section{INTRODUCTION}

In recent times, diverse statisticians exploered some new generated families of distributions by incorporate one or more extra shape parameter(s) to the baseline model to yield new flexible distributions. Some of the generated families are: the beta-G [1], Kumaraswamy (Kw)-G [2], Weibull-G [3], Garhy-G [4], exponentiated Weibull-G (EW-G) [5], additive Weibull-G [6], Kw Weibull-G [7], Type II half logistic- G [8] and exponentiated extended-G [9] among others.

Modelling lifetime phenomena is an important issue in many scientific fields. For a while, several classical models like exponential, Rayleigh, Weibull, gamma and Lomax distributions seemed suitable for modelling lifetime data. But as science improved, the old models were no longer to describe the new phenomena. So, in recent years, many researchers tried to the extend classical distributions and consequently many new flexible models appeared and proved to be more acceptable. Examples of these researches are the beta gamma distribution [10], the Weibull Rayleigh (WR) distribution [11] and the Marshall-Olkin extended generalized Rayleigh distribution [12].

We intend to introduce a new four-parameter model, called the EWR distribution. The EWR distribution is 
very flexible and it extends the WR model (see [11]). First, we explain how we arrived at this model. [5] introduced the EW- $G$ family of distributions. This family generates many new continuous distributions that are quite flexible for the purpose of modelling. The cumulative distribution function (cdf) of this family is defined by

$$
F(x)=\left[1-\exp \left(-\alpha\left[\frac{G(x)}{1-G(x)}\right]^{\beta}\right)\right]^{a} ; x \geq 0 ; a, \alpha, \beta>0,
$$

where $a, \beta$ are shape parameters, $\alpha>0$ is a scale parameter and $G(x)$ is the cdf of the baseline distribution. The probability density function (pdf) of the $\mathrm{EW}-G$ is

$$
f(x)=\frac{a \alpha \beta g(x)(G(x))^{\beta-1}}{(1-G(x))^{\beta+1}} \mathrm{e}^{-\alpha\left[\frac{G(x)}{1-G(x)}\right]^{\beta}}\left[1-\exp \left(-\alpha\left[\frac{G(x)}{1-G(x)}\right]^{\beta}\right)\right]^{a-1} ; x \geq 0 ; a, \alpha, \beta>0 .
$$

By employing the Rayleigh distribution (as the baseline distribution) in the above family, we arrive at a new flexible distribution, namely the EWR distribution. In the following sections, we obtain the pdf, cdf and hazard rate function (hrf) of the EWR distribution besides explain the shapes of the pdf and the hrf of this model briefly. We derive general statistical properties of the proposed distribution, including the expansions for the pdf and cdf, quantile function, moments, mean deviations, inequality measures, order statistics and characterizations. The maximum likelihood (ML) estimators are also discussed. Numerical study is created to estimate the model parameters. Censored Type II of the parameters is discussed. Two real data applications are presented. Finally, we point out the conclusion of our study.

\section{THE EXPONENTIATED WEIBULL RAYLEIGH DISTRIBUTION}

Depend on (1), a four-parameter EWR distribution is defined. The pdf and cdf of random variable (r.v.) $X$ has the Rayleigh distribution with scale parameter $\lambda$ are given by

$$
g(x ; \lambda)=2 \lambda x \mathrm{e}^{-\lambda x^{2}} ; \quad x, \lambda>0
$$

and

$$
G(x ; \lambda)=1-\mathrm{e}^{-\lambda x^{2}}
$$

Subsituting (3) into (1), we get the cdf of the EWR distribution as follows

$$
F(x ; \Psi)=\left[1-\exp \left(-\alpha\left(\mathrm{e}^{\lambda x^{2}}-1\right)^{\beta}\right)\right]^{a} ; \quad a, \alpha, \beta, \lambda>0 \quad, \quad x \geq 0,
$$

where $\Psi \equiv(a, \alpha, \beta, \lambda)$ is the parameter vector. From (2), (3) and (4), the pdf of EWR distribution is as follows

$$
f(x ; \Psi)=2 a \alpha \beta \lambda x\left[\mathrm{e}^{\lambda x^{2}}-1\right]^{\beta-1} \exp \left(\lambda x^{2}-\alpha\left(\mathrm{e}^{\lambda x^{2}}-1\right)^{\beta}\right)\left[1-\exp \left(-\alpha\left(\mathrm{e}^{\lambda x^{2}}-1\right)^{\beta}\right)\right]^{a-1}, x \geq 0 .
$$


We write $X \sim \operatorname{EWR}(a, \alpha, \beta, \lambda)$ if $X$ has pdf (6). Specified sub-models of the EWR distribution are added in Table 1.

Table 1. The special sub-models of the EWR distribution

\begin{tabular}{|l|l|l|l|l|l|l|l|}
\hline & Model & $a$ & $\alpha$ & $\beta$ & $\lambda$ & Distribution Function & Authors \\
\hline $\mathbf{1}$ & $\begin{array}{l}\text { Exponentaited } \\
\text { exponential } \\
\text { Rayleigh }\end{array}$ & - & - & 1 & - & $F(x)=\left[1-\exp \left(-\alpha\left(\mathrm{e}^{\lambda x^{2}}-1\right)\right)\right]^{a}$ & \\
\hline $\mathbf{2}$ & $\begin{array}{l}\text { Exponentaited } \\
\text { Rayleigh Rayleigh } \\
\text { Distribution }\end{array}$ & - & - & 2 & - & $F(x)=\left[1-\exp \left(-\alpha\left(\mathrm{e}^{\lambda x^{2}}-1\right)^{2}\right)\right]^{a}$ & \\
\hline $\mathbf{3}$ & Weibull Rayleigh & 1 & - & - & - & $F(x)=1-\exp \left(-\alpha\left(\mathrm{e}^{\lambda x^{2}}-1\right)^{\beta}\right)$ & {$[11]$} \\
\hline $\mathbf{4}$ & $\begin{array}{l}\text { Exponential } \\
\text { Rayleigh }\end{array}$ & 1 & - & 1 & - & $F(x)=1-\exp \left(-\alpha\left(\mathrm{e}^{\lambda x^{2}}-1\right)\right)$ & \\
\hline $\mathbf{5}$ & Rayleigh Rayleigh & 1 & - & 2 & - & $F(x)=1-\exp \left(-\alpha\left(\mathrm{e}^{\lambda x^{2}}-1\right)^{2}\right)$ & \\
\hline
\end{tabular}

The survival, hrf and reversed hrf of the EWR distribution, respectively, are

$$
\begin{aligned}
& R(x ; \Psi)=1-\left[1-\exp \left(-\alpha\left(\mathrm{e}^{\lambda x^{2}}-1\right)^{\beta}\right)\right]^{a}, \\
& h(x ; \Psi)=\frac{2 a \alpha \beta \lambda x\left[\mathrm{e}^{\lambda x^{2}}-1\right]^{\beta-1} \exp \left(\lambda x^{2}-\alpha\left(\mathrm{e}^{\lambda x^{2}}-1\right)^{\beta}\right)\left[1-\exp \left(-\alpha\left(\mathrm{e}^{\lambda x^{2}}-1\right)^{\beta}\right)\right]^{a-1}}{1-\left[1-\exp \left(-\alpha\left(\mathrm{e}^{\lambda x^{2}}-1\right)^{\beta}\right)\right]^{a}},
\end{aligned}
$$

and

$$
\tau(x ; \Psi)=\frac{2 a \alpha \beta \lambda x\left[\mathrm{e}^{\lambda x^{2}}-1\right]^{\beta-1} \exp \left(\lambda x^{2}-\alpha\left(\mathrm{e}^{\lambda x^{2}}-1\right)^{\beta}\right)}{1-\exp \left(-\alpha\left(\mathrm{e}^{\lambda x^{2}}-1\right)^{\beta}\right)} .
$$

The pdf and hrf plots of the EWR distribution at $\alpha=\lambda=1$ and for certain values of $a$ and $\beta$ are clarified in Figures 1 and 2, respectively. 

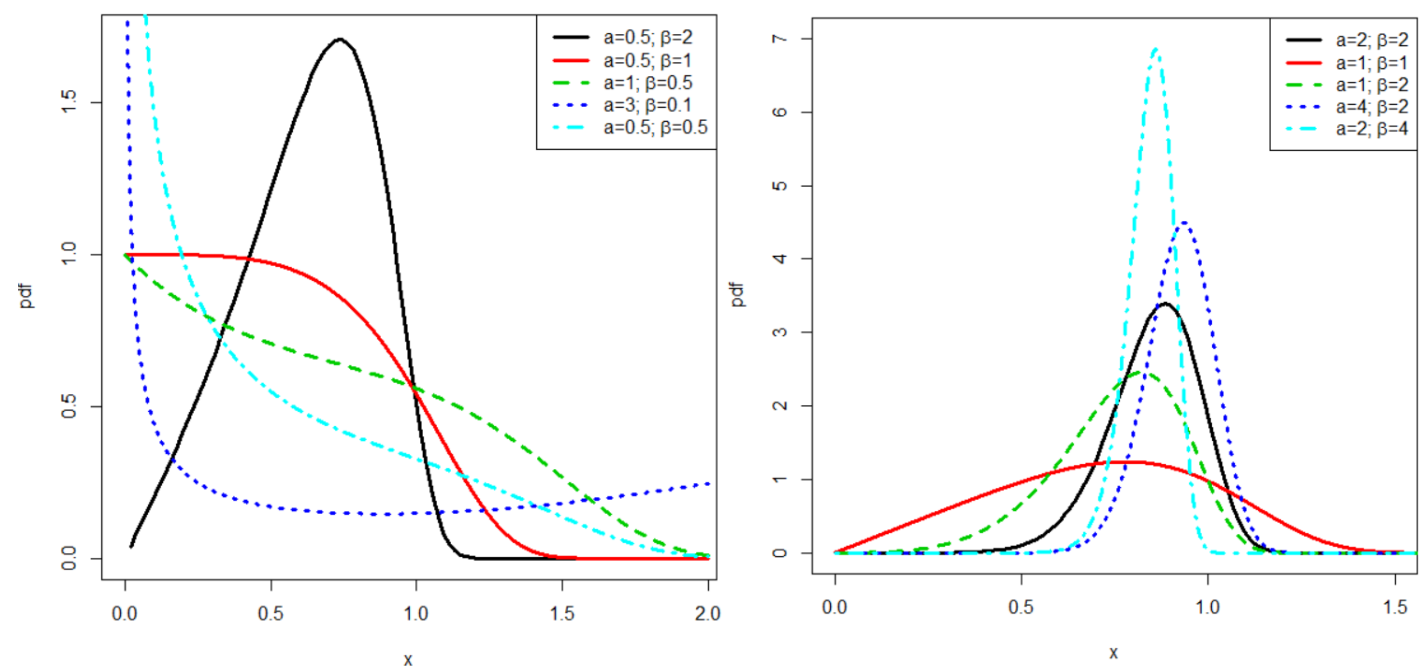

Figure 1. The pdf plots of the EWR distribution for selected parameter values at $\alpha=\lambda=1$
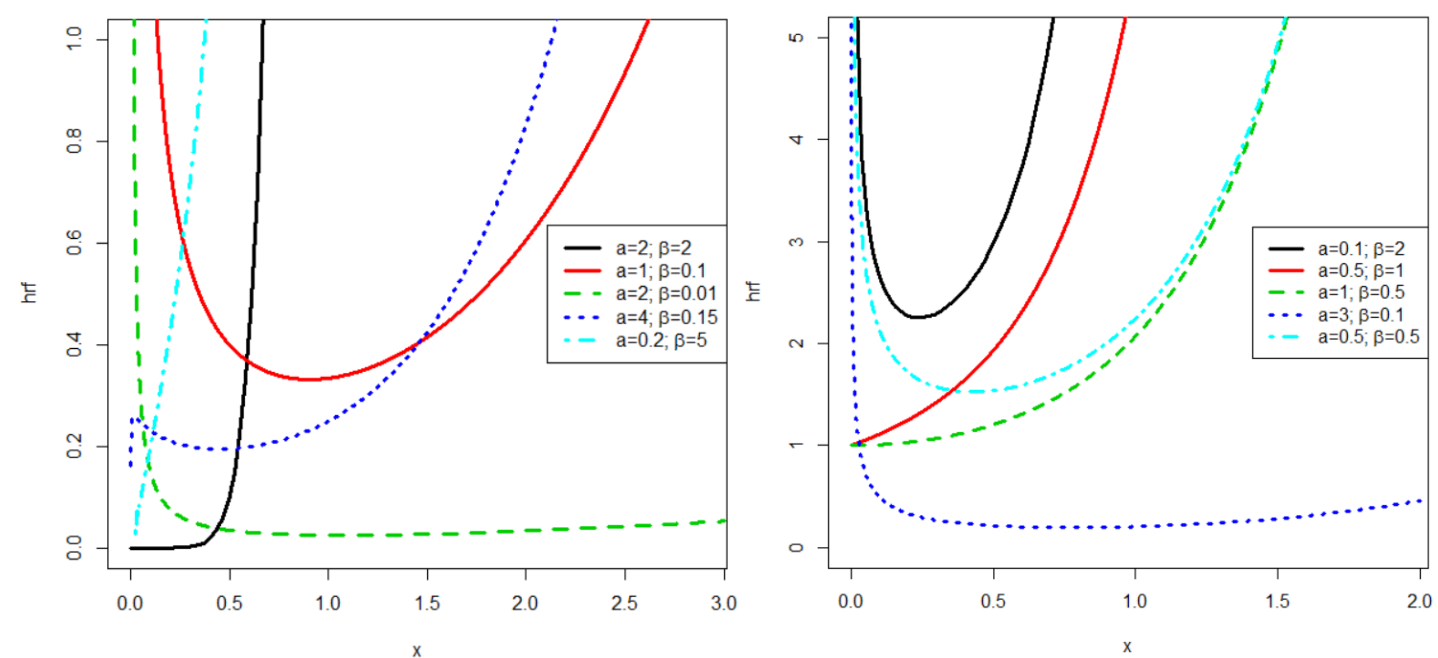

Figure 2. The hrf plots of the EWR distribution for selected parameter values at $\alpha=\lambda=1$

From Figure 2, we observe that the hrf is increasing and bathtub shaped, showing its flexibility in modelling lifetime data sets. We note that in life phenomena, we normally encounter bathtub shaped hazard rates. We also discovered an interesting case when $a=4$ and $\beta=0.15$ for which the hrf is increasing-decreasingincreasing.

\section{MAIN PROPERTIES}

Part of the properties of the EWR distribution, in this section, is explored.

\subsection{Important Expansions}

Representations of the pdf and cdf of EWR distribution are provided. First, we consider the following generalized binomial expansion,

$$
(1-h)^{d}=\sum_{i=0}^{\infty}(-1)^{i}\left(\begin{array}{l}
d \\
i
\end{array}\right) h^{i}, \quad|h|<1 .
$$

The expansion (7) stops at the $(d+1)^{\text {th }}$ term if $d$ is a positive integer value. 
Now, by applying (7), we find an expansion for the cdf raised to the power $m$, where $m$ is an integer, namely $[F(x ; \Psi)]^{m}$. We have

$$
[F(x ; \Psi)]^{m}=\sum_{q=0}^{\infty}(-1)^{q}\left(\begin{array}{c}
a m \\
q
\end{array}\right) \mathrm{e}^{-\alpha q\left(\mathrm{e}^{\lambda x^{2}}-1\right)^{\beta}} .
$$

Using power series expansion for the exponential function and the identity $\mathrm{e}^{\lambda x^{2}}-1=\left(1-\mathrm{e}^{-\lambda x^{2}}\right) \mathrm{e}^{\lambda x^{2}}$, we get

$$
\begin{aligned}
{[F(x ; \Psi)]^{m} } & =\sum_{q, w=0}^{\infty}\left(\begin{array}{c}
a m \\
q
\end{array}\right) \frac{(-1)^{q+w}(\alpha q)^{w}}{w !}\left[\mathrm{e}^{\lambda x^{2}}-1\right]^{\beta w} \\
& =\sum_{q, w=0}^{\infty}\left(\begin{array}{c}
a m \\
q
\end{array}\right) \frac{(-1)^{q+w}(\alpha q)^{w}}{w !}\left[1-\mathrm{e}^{-\lambda x^{2}}\right]^{\beta w} \mathrm{e}^{\lambda \beta w x^{2}} .
\end{aligned}
$$

Again, using (7) and the identity $\left(\begin{array}{c}-r \\ k\end{array}\right)=\left(\begin{array}{c}r+k-1 \\ k\end{array}\right)(-1)^{k}$, we have

$$
\mathrm{e}^{\lambda \beta w x^{2}}=\left(1-\left(1-\mathrm{e}^{-\lambda x^{2}}\right)\right)^{-\beta w}=\sum_{j=0}^{\infty}\left(\begin{array}{c}
\beta w+j-1 \\
j
\end{array}\right)\left(1-\mathrm{e}^{-\lambda x^{2}}\right)^{j} .
$$

Thus, $[F(x ; \Psi)]^{m}$ is written as

$$
[F(x ; \Psi)]^{m}=\sum_{k=0}^{\infty} \eta_{k, m} \mathrm{e}^{-k \lambda x^{2}}
$$

where

$$
\eta_{k, m}=\sum_{q, w, j=0}^{\infty} \frac{(-1)^{q+w+k}(\alpha q)^{w}}{w !}\left(\begin{array}{c}
a m \\
q
\end{array}\right)\left(\begin{array}{c}
\beta w+j-1 \\
j
\end{array}\right)\left(\begin{array}{c}
\beta w+j \\
k
\end{array}\right) .
$$

For $m=1$, we have the expansion for the cdf. Diffrentiating (9) with respect to $x$ when $m=1$, the pdf of the EWR will be as follows

$$
f(x ; \Psi)=2 \lambda \sum_{k=1}^{\infty} \eta_{k, 1} k x \mathrm{e}^{-k \lambda x^{2}}
$$

From (11), we see that the density of the EWR distribution is a linear combination of Rayleigh densities. Next, we find an expansion for $[f(x ; \Psi)]^{\varepsilon}, \quad \varepsilon$ is a positive number. Note that 
$[f(x ; \Psi)]^{\varepsilon}=(2 a \alpha \beta \lambda x)^{\varepsilon}\left[\mathrm{e}^{\lambda x^{2}}-1\right]^{(\beta-1) \varepsilon} \exp \left(\lambda \varepsilon x^{2}-\alpha \varepsilon\left(\mathrm{e}^{\lambda x^{2}}-1\right)^{\beta}\right)\left[1-\exp \left(-\alpha\left(\mathrm{e}^{\lambda x^{2}}-1\right)^{\beta}\right)\right]^{(a-1) \varepsilon}$.

Using (7) and exponential expansion, we have

$$
\begin{aligned}
{[f(x ; \Psi)]^{\varepsilon}=} & (2 a \alpha \beta \lambda x)^{\varepsilon}\left[\mathrm{e}^{\lambda x^{2}}-1\right]^{(\beta-1) \varepsilon} \mathrm{e}^{\lambda \varepsilon x^{2}} \sum_{q=0}^{\infty}(-1)^{q}\left(\begin{array}{c}
(a-1) \varepsilon \\
q
\end{array}\right) \mathrm{e}^{-(q+\varepsilon) \alpha\left(\mathrm{e}^{\lambda x^{2}}-1\right)^{\beta}} \\
& =(2 a \alpha \beta \lambda x)^{\varepsilon} \mathrm{e}^{\lambda \varepsilon x^{2}} \sum_{j=0}^{\infty} \sum_{q=0}^{\infty} \frac{(-1)^{j+q}[\alpha(q+\varepsilon)]^{j}}{j !}\left(\begin{array}{c}
(a-1) \varepsilon \\
q
\end{array}\right)\left(\mathrm{e}^{\left.\lambda x^{2}-1\right)^{\beta(j+\varepsilon)-\varepsilon}} .\right.
\end{aligned}
$$

Again, using identity $\mathrm{e}^{\lambda x^{2}}-1=\left(1-\mathrm{e}^{-\lambda x^{2}}\right) \mathrm{e}^{\lambda x^{2}}$, we have

$$
[f(x ; \Psi)]^{\varepsilon}=(2 a \alpha \beta \lambda x)^{\varepsilon} \mathrm{e}^{-\lambda \varepsilon x^{2}} \sum_{q, j=0}^{\infty} \frac{(-1)^{j+q}[\alpha(q+\varepsilon)]^{j}}{j !}\left(\begin{array}{c}
(a-1) \varepsilon \\
q
\end{array}\right)\left(1-\mathrm{e}^{-\lambda x^{2}}\right)^{\beta(j+\varepsilon)-\varepsilon} \mathrm{e}^{\lambda[\beta(j+\varepsilon)+\varepsilon] x^{2}}
$$

Considering (8) and using (7), we get

$$
\begin{aligned}
{[f(x ; \Psi)]^{\delta} } & =(2 a \alpha \beta \lambda x)^{\varepsilon} \mathrm{e}^{-\lambda \varepsilon x^{2}} \sum_{q, j, w=0}^{\infty} \frac{(-1)^{j+q}[\alpha(q+\varepsilon)]^{j}}{j !}\left(\begin{array}{c}
(a-1) \varepsilon \\
q
\end{array}\right)\left(\begin{array}{c}
\beta(j+\varepsilon)+\varepsilon+w-1 \\
w
\end{array}\right)\left(1-\mathrm{e}^{-\lambda x^{2}}\right)^{\beta(j+\varepsilon)-\varepsilon+w} \\
& =(2 a \alpha \beta \lambda x)^{\varepsilon} \sum_{q, j, w, k=0}^{\infty} \frac{(-1)^{j+q+k}[\alpha(q+\varepsilon)]^{j}}{j !}\left(\begin{array}{c}
(a-1) \varepsilon \\
q
\end{array}\right)\left(\begin{array}{c}
\beta(j+\varepsilon)+\varepsilon+w-1 \\
w
\end{array}\right)\left(\begin{array}{c}
\beta(j+\varepsilon)-\varepsilon+w \\
k
\end{array}\right) \mathrm{e}^{-(k+1) \lambda x^{2}} .
\end{aligned}
$$

From the above relations, we arrive at

$$
[f(x ; \Psi)]^{\varepsilon}=(2 \lambda x)^{\varepsilon} \sum_{k=1}^{\infty} \eta_{k, \varepsilon}^{*} \mathrm{e}^{-k \lambda x^{2}}
$$

where

$$
\eta_{k, \varepsilon}^{*}=(a \alpha \beta)^{\varepsilon} \sum_{q, j, w=0}^{\infty} \frac{(-1)^{j+q+k-1}[\alpha(q+\varepsilon)]^{j}}{j !}\left(\begin{array}{c}
(a-1) \varepsilon \\
q
\end{array}\right)\left(\begin{array}{c}
\beta(j+\varepsilon)+\varepsilon+w-1 \\
w
\end{array}\right)\left(\begin{array}{c}
\beta(j+\varepsilon)-\varepsilon+w \\
k-1
\end{array}\right) .
$$

We can use (12) as an alternative expansion for the pdf of the EWR distribution after setting $\varepsilon=1$.

\subsection{Quantile Function}

The quantile function, say $Q(u)=F^{-1}(u)$, of $X$ is obtained by inverting (5). So we have

$$
Q(u)=\sqrt{\frac{1}{\lambda} \ln \left\{1+\left(\frac{-1}{\alpha} \ln \left(1-u^{\frac{1}{a}}\right)\right)^{\frac{1}{\beta}}\right\}}
$$


where $0<u<1$. Note that if $\mathrm{U}$ is a uniform $(0,1)$, then $Q(\mathrm{U})$ is an EWR r.v. Therefore, one can simulate numbers from EWR distribution by using (14). Given $u=0.5$ in (14), the median of EWR model is obtained as follows

$$
M=Q(0.5)=\sqrt{\frac{1}{\lambda} \ln \left\{1+\left(\frac{-1}{\alpha} \ln \left(1-0.5^{\frac{1}{a}}\right)\right)^{\frac{1}{\beta}}\right\}}
$$

Furthermore, based on quantile measures, the variability analysis of the skewness and kurtosis on the shape parameters $a$ and $\beta$ is inspected. The Bowley (B) skewness (see [13]), is defined by

$$
\mathrm{B}=\frac{X_{\frac{3}{4}}-2 X_{\frac{1}{2}}+X_{\frac{1}{4}}}{X_{\frac{3}{4}}-X_{\frac{1}{4}}} .
$$

The Moors (MO) kurtosis, (see [14]), is defined by

$$
\mathrm{MO}=\frac{X_{\frac{7}{8}}-X_{\frac{5}{8}}+X_{\frac{3}{8}}-X_{\frac{1}{8}}}{X_{\frac{6}{8}}-X_{\frac{2}{8}}} .
$$

The B and MO measures do not rely on the moments of the distribution and are nearly insensitive to outliers. Plots of the $\mathrm{B}$ and MO for certain values of $a$ as function of $\beta$ and for certain choices of $\beta$ as function of $a$ are provided in Figures 3 and 4.

(a)

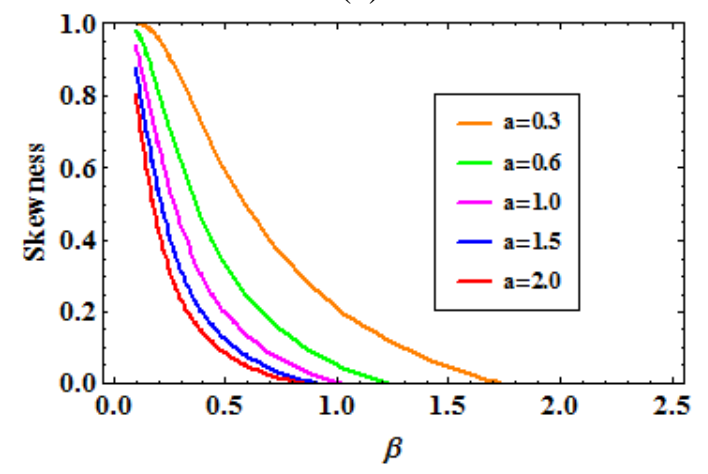

(b)

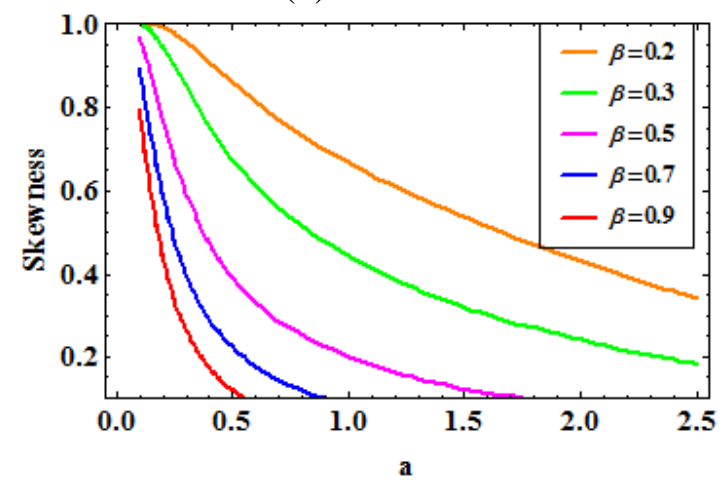

Figure 3. B skewness of the EWR distribution. (a) As function of $\beta$ for certain values of a ( $b$ ) As function of a for certain values of $\beta$ at $\alpha=\lambda=2$ 
(a)

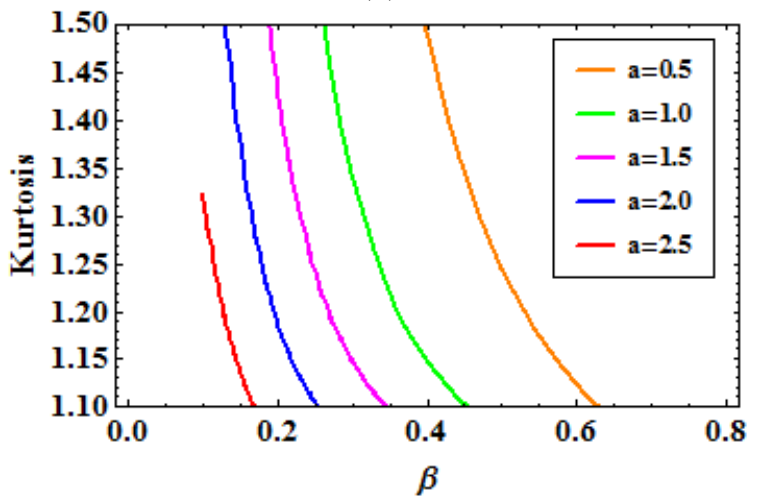

(b)

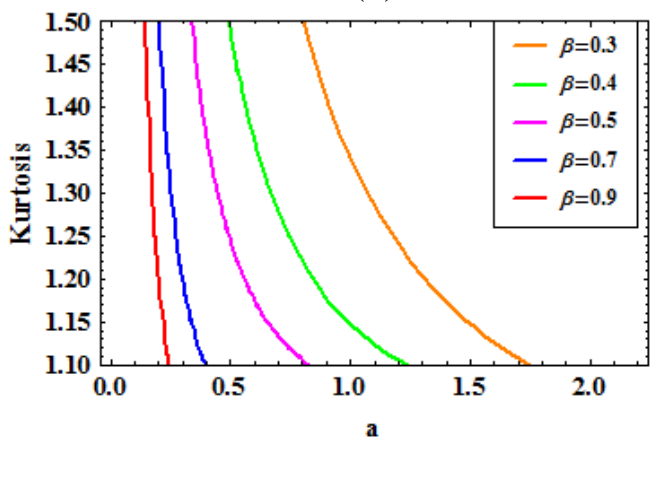

Figure 4. MO kurtosis of the EWR distribution. (a) As function of $\beta$ for certain values of a (b) As function of a for certain values of $\beta$ at $\alpha=\lambda=2$

\subsection{Moments}

The $r^{\text {th }}$ moment of EWR model is given by

$$
\mu_{r}^{\prime}=\int_{0}^{\infty} x^{r} f(x ; \Psi) \mathrm{d} x=\Gamma\left(\frac{r}{2}+1\right) \sum_{k=1}^{\infty} \frac{\eta_{k, 1}}{(k \lambda)^{\frac{r}{2}}},
$$

where $\eta_{k, m}$ is given in (10) and $\Gamma($.$) is the gamma function. In addition, the moment generating function of$ EWR model is given by

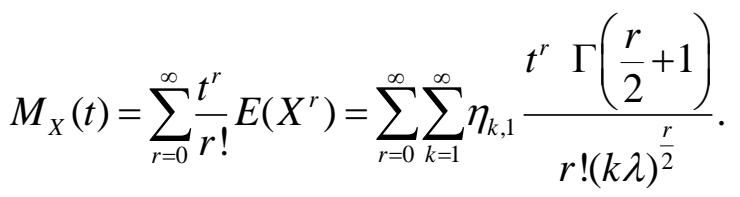

\subsection{Mean Deviations}

Two important dispersion measures are the mean deviation from the mean $(\mu)$ and the mean deviation from the median $(M)$ denoted by $\delta_{1}$ and $\delta_{2}$, are defined, respectively, by

$\delta_{1}=\int_{0}^{\infty}|x-\mu| f(x, \Psi) \mathrm{d} x \quad$ and $\quad \delta_{2}=\int_{0}^{\infty}|x-M| f(x, \Psi) \mathrm{d} x$

First, we obtain the incomplete moments, denoted by $\varphi_{s}(t)$, of the EWR distribution as follows, where $\varphi_{s}(t)$, defined by

$\varphi_{s}(t)=\int_{0}^{t} x^{s} f(x) \mathrm{d} x$.

Using (11), $\varphi_{s}(t)$ will be as given 


$$
\varphi_{s}(t)=2 \lambda \sum_{k=1}^{\infty} \eta_{k, 1} k \int_{0}^{t} x^{s+1} \mathrm{e}^{-k \lambda x^{2}} \mathrm{~d} x=\sum_{k=1}^{\infty} \frac{\eta_{k, 1}}{(k \lambda)^{\frac{s}{2}}} \Gamma\left(\frac{s}{2}+1, k \lambda t^{2}\right)
$$

where $\Gamma(s, t)=\int_{0}^{t} x^{s-1} \mathrm{e}^{-x} \mathrm{~d} x$ is the lower incomplete gamma function.

Now, the mean deviations from $\mu$ and the mean deviation from $\mathrm{M}$, respectively, are

$$
\delta_{1}=2 \mu F(\mu)-2 \varphi_{1}(\mu) \quad \text { and } \quad \delta_{2}=\mu-2 \varphi_{1}(M) \text {, }
$$

where $\varphi_{1}(t)$ and $M$ are given, in (17) and (15) respectively.

\subsection{Residual Life Function}

The $n^{\text {th }}$ moment of the residual life (see [15-17]), is specified by

$$
m_{n}(t)=E\left[(X-t)^{n} \mid X>t\right]=\frac{1}{R(t)} \int_{t}^{\infty}(x-t)^{n} f(x) \mathrm{d} x
$$

Employing the pdf (11) and binomial expansion for $(x-t)^{n}$, then $m_{n}(t)$ will be

$$
m_{n}(t)=\frac{1}{R(t ; \Psi)} \sum_{k=1}^{\infty} \sum_{r=0}^{n} \frac{(-t)^{n-r} \eta_{k, 1}}{(k \lambda)^{\frac{r}{2}}}\left(\begin{array}{l}
n \\
r
\end{array}\right) \gamma\left(\frac{r}{2}+1, k \lambda t^{2}\right),
$$

where $\gamma(s, t)$ is the upper incomplete gamma function. For, $n=1$, in (18), we obtain the so called mean residual life (MRL). The MRL as pointed out is the expected supplemantry life length of an item that is alive at age $t$. Hence, if $n=1$ in (18), we get the MRL of EWR model.

On the other hand, $n^{\text {th }}$ order moment of reversed residual life (RRL) is

$$
M_{n}(t)=E\left[(t-X)^{n} \mid X \leq t\right]=\frac{1}{F(t)} \int_{0}^{t}(t-x)^{n} f(x) \mathrm{d} x
$$

So, the moments of RRL for EWR model is

$$
M_{n}(t)=\frac{1}{F(t, \Psi)} \sum_{k=1}^{\infty} \sum_{r=0}^{n} \frac{(-1)^{r} t^{n-r} \eta_{k, 1}}{(k \lambda)^{\frac{r}{2}}}\left(\begin{array}{l}
n \\
r
\end{array}\right) \Gamma\left(\frac{r}{2}+1, k \lambda t^{2}\right) .
$$

\subsection{Inequality Measures}

The Lorenz, Bonferroni and Zenga curves (see [18]) are obtained, respectively, as follows 


$$
\begin{gathered}
L_{F}(x)=\frac{\varphi_{1}(x)}{E(X)}=\frac{2 \sum_{k=1}^{\infty} \frac{\eta_{k, 1}}{(k \lambda)^{\frac{3}{2}}} \Gamma\left(\frac{3}{2}, k \lambda x^{2}\right)}{\sqrt{\pi} \sum_{k=1}^{\infty} \frac{\eta_{k, 1}}{(k \lambda)^{\frac{3}{2}}}}, \\
B_{F}(x)=\frac{L_{F}(x)}{F(x ; \Psi)}=\frac{2 \sum_{k=1}^{\infty} \frac{\eta_{k, 1}}{(k \lambda)^{\frac{3}{2}}} \Gamma\left(\frac{3}{2}, k \lambda x^{2}\right)}{\sqrt{\pi} \sum_{q=0}^{\infty} \sum_{k=1}^{\infty} \frac{\eta_{k, 1} \eta_{q, 1}}{(k \lambda)^{\frac{3}{2}}} \mathrm{e}^{-q \lambda x^{2}}},
\end{gathered}
$$

and

$$
A_{F}(x)=1-\frac{\mu^{-}(x)}{\mu^{+}(x)}
$$

where

$\mu^{-}(x)=\frac{\varphi_{1}(x)}{F(x ; \Psi)}=\frac{\sum_{k=1}^{\infty} \frac{\eta_{k, 1}}{(k \lambda)^{\frac{3}{2}}} \Gamma\left(\frac{3}{2}, k \lambda x^{2}\right)}{\sum_{k=0}^{\infty} \eta_{k, 1} \mathrm{e}^{-k \lambda x^{2}}}$,

and

$$
\mu^{+}(x)==\frac{\int_{x}^{\infty} t f(t, \Psi) \mathrm{d} t}{R(x ; \Psi)}=\frac{\sum_{k=1}^{\infty} \frac{\eta_{k, 1}}{(k \lambda)^{\frac{3}{2}}} \gamma\left(\frac{3}{2}, k \lambda x^{2}\right)}{1-\sum_{k=0}^{\infty} \eta_{k, 1} \mathrm{e}^{-k \lambda x^{2}}} .
$$

\subsection{Rényi and $\varepsilon$-Entropies}

For a r.v. $X$, the Rényi entropy is given by

$$
I_{\varepsilon}(X)=(1-\varepsilon)^{-1} \log \int_{0}^{\infty} f(x)^{\varepsilon} \mathrm{d} x, \quad \varepsilon>0 \text { and } \varepsilon \neq 1
$$

Using (12), the Rényi entropy of EWR distribution is given by

$$
I_{\varepsilon}(X)=(1-\varepsilon)^{-1} \log \left[(2 \lambda x)^{\varepsilon} \sum_{k=1}^{\infty} \eta_{k, \varepsilon}^{*} \mathrm{e}^{-k \lambda x^{2}}\right] \text {, }
$$


where $\eta_{k, \varepsilon}^{*}$ is defined in (13). Furthermore, the $\varepsilon$-entropy is defined by

$$
H_{\varepsilon}(X)=(1-\varepsilon)^{-1} \log \left(1-\int_{0}^{\infty} f(x)^{\varepsilon} \mathrm{d} x\right), \quad \varepsilon>0 \text { and } \varepsilon \neq 1 .
$$

Therefore, the $\varepsilon$-entropy of EWR distribution is given by

$$
H_{\varepsilon}(X)=(1-\varepsilon)^{-1} \log \left\{1-(2 \lambda x)^{\varepsilon} \sum_{k=1}^{\infty} \eta_{k, \varepsilon}^{*} \mathrm{e}^{-k \lambda x^{2}}\right\} \text {. }
$$

\subsection{Order Statistics}

Let $X_{(1)}, \ldots, X_{(n)}$ be the order statistics (Os) of a random sample of size $n$ from EWR distribution, then the pdf of the $s^{\text {th }}$ Os, is given ( see [19]) by

$$
f_{X_{(s)}}(x)=\frac{f(x)}{B(s, n-s+1)} \sum_{v=0}^{n-s}(-1)^{v}\left(\begin{array}{c}
n-s \\
v
\end{array}\right)[F(x)]^{v+s-1},
$$

where $B(.,$.$) is the beta function. By substituting (9) and (11) into (19) and replacing m$ with $v+s-1$, we have

$$
f_{X_{(s)}}(x)=\frac{2 \lambda x}{B(s, n-s+1)} \sum_{v=0}^{n-s} \sum_{k_{1}=1}^{\infty} \sum_{k_{2}=0}^{\infty} \zeta_{v, s, k_{1}, k_{2}} \mathrm{e}^{-\lambda\left(k_{1}+k_{2}\right) x^{2}}
$$

where $\zeta_{v, s, k_{1}, k_{2}}=k_{1}(-1)^{v}\left(\begin{array}{c}n-s \\ v\end{array}\right) \eta_{k_{2}, v+s-1} \eta_{k_{1}, 1}$

In addition, from (20), the $r^{\text {th }}$ moment of $X_{(\mathrm{s})}$ is given by

$$
E\left(X_{(s)}^{r}\right)=\frac{\Gamma((r / 2)+1)}{\lambda^{\frac{r}{2}} B(s, n-s+1)} \sum_{v=0}^{n-s} \sum_{k_{1}=1}^{\infty} \sum_{k_{2}=0}^{\infty} \frac{\zeta_{v, s, k_{1}, k_{2}}}{\left(k_{1}+k_{2}\right)^{\frac{r}{2}+1}} .
$$

\section{CHARACTERIZATIONS}

Here, we provide certain characterizations of EWR distribution. However, these characterizations (CHs) are based on: (i) a relation between two truncated moments; (ii) the hrf ; (iii) the reversed hrf and (iv) conditional expectation of a function of the r.v. One of the benefits of $\mathrm{CH}$ (i) is that the cdf does not required to be in closed form. $\mathrm{CHs}$ in our study are provided in the following subsections.

\subsection{Characterizations via Ratio of Truncated Moments}

We offer CHs of EWR distribution in terms of a simple relationship between two truncated moments. This $\mathrm{CH}$ result employs a theorem due to [20] (see Theorem 1- Appendix A). Note that the result holds also when the interval $\mathrm{H}$ is not closed. However, as previously mentioned, it can be also applied when the cdf does not have a closed form. According to [21], this $\mathrm{CH}$ is stable in the sense of weak convergence.

Proposition 4.1. Let $X: \Omega \rightarrow(0, \infty)$ is a continuous r.v. and let 


$$
q_{1}(x)=\left[1-\exp \left(-\alpha\left(\mathrm{e}^{\lambda x^{2}}-1\right)^{\beta}\right)\right]^{1-\alpha}, \text { and } q_{2}(x)=q_{1}(x) \exp \left(-\alpha\left(\mathrm{e}^{\lambda x^{2}}-1\right)^{\beta}\right)
$$

for $x>0$. The r.v. $X$ has pdf (6) if and only if the function $\eta$ defined in Theorem 1 has the form $\eta(x)=\frac{1}{2} \exp \left(-\alpha\left(\mathrm{e}^{\lambda x^{2}}-1\right)^{\beta}\right), \quad x>0$.

Proof. Let $X$ be a r.v. with pdf (6), then

$$
\begin{aligned}
& (1-\mathrm{F}(x)) \mathrm{E}\left[q_{1}(X) \mid X \geq x\right]=a \exp \left(-\alpha\left(\mathrm{e}^{\lambda x^{2}}-1\right)^{\beta}\right), \quad x>0, \\
& (1-\mathrm{F}(x)) \mathrm{E}\left[q_{2}(X) \mid X \geq x\right]=\frac{1}{2} \exp \left(-2 \alpha\left(\mathrm{e}^{\lambda x^{2}}-1\right)^{\beta}\right), \quad x>0 .
\end{aligned}
$$

and

$$
\eta(x) q_{1}(x)-q_{2}(x)=-\frac{1}{2} q_{1}(x) \exp \left(-\alpha\left(\mathrm{e}^{\lambda x^{2}}-1\right)^{\beta}\right)<0 \text { for } x>0 .
$$

Conversely, if $\eta$ is given as above, then

$s^{\prime}(x)=\frac{\eta^{\prime}(x) q_{1}(x)}{\eta(x) q_{1}(x)-q_{2}(x)}=\alpha \beta x e^{\lambda x^{2}}\left(e^{\lambda x^{2}}-1\right)^{\beta-1} \quad x>0$.

And

$s^{\prime}(x)=\alpha\left(e^{\lambda x^{2}}-1\right)^{\beta-1} \quad x>0$.

According to Theorem 1, then $X$ has pdf (6).

Corollary 4.1. Let $X: \Omega \rightarrow(0, \infty)$ be a continuous r.v. and let $q_{1}(x)$ be as in Proposition 4.1. The pdf of $X$ is (6) if and only if there exist functions $q_{2}(x)$ and $\eta$ defined in Theorem 1 verifying the differential equation $\frac{\eta^{\prime}(x) q_{1}(x)}{\eta(x) q_{1}(x)-q_{2}(x)}=\alpha \beta x e^{\lambda x^{2}}\left(e^{\lambda x^{2}}-1\right)^{\beta-1} \quad x>0$.

The general solution of the differential equation in Corollary 4.1 is

$$
\eta(x)=\exp \left(-\alpha\left(\mathrm{e}^{\lambda x^{2}}-1\right)^{\beta}\right)\left[\begin{array}{l}
\alpha \beta x e^{\lambda x^{2}}\left(e^{\lambda x^{2}}-1\right)^{\beta-1} \times \\
\exp \left(-\alpha\left(\mathrm{e}^{\lambda x^{2}}-1\right)^{\beta}\right)\left(q_{1}(x)\right)^{-1} q_{2}(x)+\mathrm{D}
\end{array}\right],
$$

where $\mathrm{D}$ be a constant. Note that a set of functions verifying the above differential equation is provided in Proposition 4.1 for $\mathrm{D}=0$. Also, it pay attension to note that there are other triplets $\left(q_{1}(x), q_{2}(x), \eta\right)$ verifying the conditions of Theorem 1 .

\subsection{Characterization via Hazard Function}


It is known that the hrf, $h_{F}$, of a twice differentiable distribution function, $F$, verifies the first order differential equation

$$
\frac{f^{\prime}(x)}{f(x)}=\frac{h_{F}^{\prime}}{h_{F}}-h_{F}(x) .
$$

For various univariate continuous distributions, this is the only $\mathrm{CH}$ available in terms of the hrf. The following $\mathrm{CH}$ create a non-trivial $\mathrm{CH}$ of EWR distribution, when $a=1$, which is not of the above trivial form.

Proposition 4.2. Let $X: \Omega \rightarrow(0, \infty)$ be a continuous r.v. The pdf of $X$ for $a=1$, is (6) if and only if its hrf $h_{F}(x)$ verifies the differential equation

$$
h_{F}^{\prime}(x)-x^{-1} h_{F}^{\prime}(x)=4 \alpha \beta \lambda^{2} x^{2} e^{\lambda x^{2}}\left(e^{\lambda x^{2}}-1\right)^{\beta-1}\left(2 e^{\lambda x^{2}}-1\right), \quad x>0 .
$$

Proof. If $X$ has pdf (6), then the above differential equation holds, then for $x>0$,

$$
\frac{d}{d x}\left\{x^{-1} h_{F}(x)\right\}=2 \alpha \beta \lambda \frac{d}{d x}\left\{e^{\lambda x^{2}}\left(e^{\lambda x^{2}}-1\right)^{\beta-1}\right\}
$$

from which, we obtain

$$
h_{F}(x)=2 \alpha \beta \lambda x e^{\lambda x^{2}}\left(e^{\lambda x^{2}}-1\right)^{\beta-1}, x>0
$$

which is the hrf of EWR distribution for $a=1$.

\subsection{Characterizations on the basis of the Reversed hrf}

The reversed hrf, $r_{F}$, of a twice differentiable distribution function, $F$, is defined as

$r_{F}(x)=\frac{f(x)}{F(x)}, x \in$ support of $F$

Proposition 4.3. Let $X: \Omega \rightarrow(0, \infty)$ be a continuous r.v. The r.v. $X$ has pdf (6) if and only if its reversed hrf $r_{F}$ verifies the following differential equation

$$
r_{F}^{\prime}(x)-x^{-1} r_{F}(x)=2 \alpha \beta \lambda x \frac{d}{d x}\left\{\frac{\left(e^{\lambda x^{2}}-1\right)^{\beta-1} \exp \left\{\lambda x^{2}-\alpha\left(e^{\lambda x^{2}}-1\right)^{\beta}\right\}}{1-\exp \left\{-\alpha\left(e^{\lambda x^{2}}-1\right)^{\beta}\right\}}\right\}, \quad x>0 .
$$

Proof: If $X$ has pdf (6), then clearly the above differential equation holds. That is 
$\frac{d}{d x}\left\{x^{-1} r_{F}(x)\right\}=2 a \alpha \beta \lambda \frac{d}{d x}\left\{\frac{\left(e^{\lambda x^{2}}-1\right)^{\beta-1} \exp \left\{\lambda x^{2}-\alpha\left(e^{\lambda x^{2}}-1\right)^{\beta}\right\}}{1-\exp \left\{-\alpha\left(e^{\lambda x^{2}}-1\right)^{\beta}\right\}}\right\}$

from which, we have, for $x>0$

$r_{F}(x)=\frac{2 a \alpha \beta \lambda x\left(e^{\lambda x^{2}}-1\right)^{\beta-1} \exp \left\{\lambda x^{2}-\alpha\left(e^{\lambda x^{2}}-1\right)^{\beta}\right\}}{1-\exp \left\{-\alpha\left(e^{\lambda x^{2}}-1\right)^{\beta}\right\}}$.

\subsection{Characterization via the Conditional Expectation of Certain Functions of the Random Variable}

Here, we utilize a single function $\psi$ of $X$ and characterize the distribution of $X$ in terms of the truncated moment of $\psi(x)$. Proposition 4.4 is used to characterize EWR distribution, clearly, this proposition has previously appeared in [22].

Proposition 4.4. Let $X: \Omega \rightarrow(0, \infty)$ be a continuous r.v. with cdf $F$. Let $\psi(x)$ be a differentiable function on $(c, e)$ with $\lim _{x \rightarrow e^{-}} \psi(x)=1$. Then, for $\delta \neq 1$,

$E[\psi(X) \mid X \leq x]=\delta \psi(x), \quad x \in(c, e)$.

Implies

$\psi(x)=(F(x))^{\frac{1}{\delta}-1}, \quad x \in(c, e)$.

Remark 4.4. For $(c, e)=(0, \infty), \psi(x)=1-\exp \left\{-\alpha\left(e^{\lambda x^{2}}-1\right)^{\beta}\right\}$ and $\delta=\frac{a}{a+1}$, Proposition 4.4 provides a $\mathrm{CH}$ of EWR distribution.

\section{MAXIMUM LIKELIHOOD ESTIMATION}

ML proedure is the commonly employed method of estimation. The estimators that are obtained based on this procedure enjoy desirable asypmtotic properties and therefore they are often utilized to obtain confidence intervals (CI) and test of statistical hypotheses. Suppose that $x_{1}, \cdots, x_{n}$ be an observed random sample from the EWR distribution with pdf (6). Then the log-likelihood function, denoted by $\ln \ell$, for the set of parameters $\Psi=(a, \alpha, \beta, \lambda)$ is

$$
\begin{aligned}
\ln \ell= & n \ln 2 a+n \ln \alpha+n \ln \beta+n \ln \lambda+\sum_{i=1}^{n} \ln \left(x_{i}\right)+(\beta-1) \sum_{i=1}^{n} \ln \left(\mathrm{e}^{\lambda x_{i}{ }^{2}}-1\right) \\
& +\lambda \sum_{i=1}^{n} x_{i}^{2}-\alpha \sum_{i=1}^{n}\left(\mathrm{e}^{\lambda x_{i}{ }^{2}}-1\right)^{\beta}+(a-1) \sum_{i=1}^{n} \ln \left[1-\exp \left[-\alpha\left(\mathrm{e}^{\lambda x_{i}{ }^{2}}-1\right)^{\beta}\right]\right] .
\end{aligned}
$$


By differentiating (21) partially with respect to unknown parameters, then, we have

$$
\begin{aligned}
& \frac{\partial \ln \ell}{\partial a}=\frac{n}{a}+\sum_{i=1}^{n} \ln \left[1-\exp \left[-\alpha\left(\mathrm{e}^{\lambda x_{i}^{2}}-1\right)^{\beta}\right]\right], \\
& \frac{\partial \ln \ell}{\partial \alpha}=\frac{n}{\alpha}-\sum_{i=1}^{n}\left(\mathrm{e}^{\lambda x_{i}^{2}}-1\right)^{\beta}+(a-1) \sum_{i=1}^{n} \frac{\left(\mathrm{e}^{\lambda x_{i}^{2}}-1\right)^{\beta} \exp \left[-\alpha\left(\mathrm{e}^{\lambda x_{i}^{2}}-1\right)^{\beta}\right]}{1-\exp \left[-\alpha\left(\mathrm{e}^{\lambda x_{i}^{2}}-1\right)^{\beta}\right]}, \\
& \frac{\partial \ln \ell}{\partial \beta}=\frac{n}{\beta}+\sum_{i=1}^{n} \ln \left(\mathrm{e}^{\lambda x_{i}^{2}}-1\right)-\alpha \sum_{i=1}^{n}\left(\mathrm{e}^{\lambda x_{i}^{2}}-1\right)^{\beta} \ln \left(\mathrm{e}^{\lambda x_{i}^{2}}-1\right) \\
& +\alpha(a-1) \sum_{i=1}^{n} \frac{\left(\mathrm{e}^{\lambda x_{i}^{2}}-1\right)^{\beta} \exp \left[-\alpha\left(\mathrm{e}^{\lambda x_{i}^{2}}-1\right)^{\beta}\right] \ln \left(\mathrm{e}^{\lambda x_{i}^{2}}-1\right)}{1-\exp \left[-\alpha\left(\mathrm{e}^{\lambda x_{i}^{2}}-1\right)^{\beta}\right]},
\end{aligned}
$$

and

$$
\begin{aligned}
\frac{\partial \ln \ell}{\partial \lambda}=\frac{n}{\lambda} & +(\beta-1) \sum_{i=1}^{n} \frac{x_{i}^{2} \mathrm{e}^{\lambda x_{i}^{2}}}{\mathrm{e}^{\lambda x_{i}^{2}}-1}+\alpha \beta(a-1) \sum_{i=1}^{n} \frac{x_{i}^{2} \mathrm{e}^{\lambda x_{i}^{2}}\left(\mathrm{e}^{\lambda x_{i}^{2}}-1\right)^{\beta-1} \mathrm{e}^{-\alpha\left(\mathrm{e}^{\lambda x_{i}^{2}}-1\right)^{\beta}}}{1-\exp \left[-\alpha\left(\mathrm{e}^{\lambda x_{i}^{2}}-1\right)^{\beta}\right]}+\sum_{i=1}^{n} x_{i}^{2} \\
& -\alpha \beta \sum_{i=1}^{n} x_{i}^{2}\left(\mathrm{e}^{\lambda x_{i}^{2}}-1\right)^{\beta-1} \mathrm{e}^{\lambda x_{i}^{2}} .
\end{aligned}
$$

The ML estimates are obtained numerically as solutions of the above nonlinear equations after setting them equal zeros. The observed information matrix is required in case of interval estimation. This $4 \times 4$ symmetric matrix, denoted by I $(\Psi)$, is derived by considering the second-order derivatives of the $\ln \ell$, with respect to the parameters. Under regularity conditions, the vector of the ML estimators of the parameters, denoted by $\hat{\Psi}^{T}=(\hat{a}, \hat{\alpha}, \hat{\beta}, \hat{\lambda})^{T}$, approximately possesses a 4-variate normal distribution with the mean $\Psi^{\mathrm{T}}$ and the variance-covariance matrix $\mathrm{I}^{-1}(\Psi)$, where $\mathrm{I}^{-1}(\Psi)$ is the inverse matrix of $\mathrm{I}(\Psi)$. This property of ML estimators can be used to obtain approximate CI for the model parameters. We note that the elements of $\mathrm{I}(\Psi)$ depend on the parameters and the ML estimators may substitute for the corresponding parameters to evaluate $\mathrm{I}(\Psi)$.

\section{SIMULATION ILLUSTRATION}

A numerical investigation is established to examine the behavior of ML estimates (MLE) for EWR model. For different sample sizes, measures like; biases and mean square errors (MSEs) are calculated to evaluate the performance of estimates. A numerical study is done using Mathematica (9) software. The steps of simulation procedure is listed as follows

- We generate 10000 from EWR distribution of sizes; $n=30,50,75$ and 100 .

- $\quad$ Certain values of parameters $(a, \alpha, \beta, \lambda)$ are chosen as Set $1=(2,2,0.5,1.5)$, Set $2=(1.5,2,0.5,1.5)$, Set $3=(2,2,0.75,1.5)$ and Set $4=(2,2,0.9,1.5)$.

- $\quad$ MLE of the parameters $\hat{a}, \hat{\alpha}, \hat{\beta}$ and $\hat{\lambda}$ are calculated for each $n$ and for all sets. 
- Calculate the biases and MSE for each $n$.

- Result outcomes are listed in Tables 2 and 3.

Table 2. Parameter estimates of EWR distribution for different sample sizes

\begin{tabular}{|c|c|c|c|c|c|c|c|}
\hline \multirow[t]{2}{*}{$n$} & Parameter & Mean & Bias & MSE & Mean & Bias & MSE \\
\hline & & \multicolumn{3}{|c|}{ Set $1=(2,2,0.5,1.5)$} & \multicolumn{3}{|c|}{ Set $2=(1.5,2,0.5 .1,5)$} \\
\hline \multirow{4}{*}{30} & $a$ & 2.0661 & 0.0661 & 0.1521 & 1.5538 & 0.0538 & 0.0881 \\
\hline & $\alpha$ & 2.1413 & 0.1413 & 0.4373 & 2.0987 & 0.0987 & 0.2603 \\
\hline & $\beta$ & 0.5307 & 0.0307 & 0.0208 & 0.5328 & 0.0328 & 0.0212 \\
\hline & $\lambda$ & 1.5525 & 0.0525 & 0.0908 & 1.5381 & 0.0381 & 0.0627 \\
\hline \multirow{4}{*}{50} & $a$ & 2.0365 & 0.0365 & 0.0893 & 1.5290 & 0.0290 & 0.0505 \\
\hline & $\alpha$ & 2.0864 & 0.0863 & 0.2112 & 2.0626 & 0.0626 & 0.1374 \\
\hline & $\beta$ & 0.5179 & 0.0179 & 0.0112 & 0.5185 & 0.0185 & 0.0111 \\
\hline & $\lambda$ & 1.5359 & 0.0359 & 0.0545 & 1.5267 & 0.0266 & 0.0380 \\
\hline \multirow{4}{*}{75} & $a$ & 2.0287 & 0.0287 & 0.0571 & 1.5195 & 0.0195 & 0.0322 \\
\hline & $\alpha$ & 2.0495 & 0.0495 & 0.1165 & 2.0367 & 0.0367 & 0.0850 \\
\hline & $\beta$ & 0.5128 & 0.0128 & 0.0069 & 0.5115 & 0.0115 & 0.0068 \\
\hline & $\lambda$ & 1.5215 & 0.0215 & 0.0344 & 1.5154 & 0.0154 & 0.0250 \\
\hline \multirow{4}{*}{$\begin{array}{l}10 \\
0\end{array}$} & $a$ & 2.0217 & 0.0217 & 0.0426 & 1.5152 & 0.0152 & 0.0232 \\
\hline & $\alpha$ & 2.0344 & 0.0344 & 0.0825 & 2.0279 & 0.0279 & 0.0611 \\
\hline & $\beta$ & 0.5093 & 0.0093 & 0.0049 & 0.5091 & 0.0091 & 0.0048 \\
\hline & $\lambda$ & 1.5154 & 0.0154 & 0.0252 & 1.5121 & 0.0121 & 0.0188 \\
\hline
\end{tabular}

Table 3. Parameter estimates of EWR distribution for different sample sizes

\begin{tabular}{|c|c|c|c|c|c|c|c|}
\hline$n$ & Parameter & Mean & Bias & MSE & Mean & Bias & MSE \\
\hline & & \multicolumn{3}{|c|}{ set $3=(2,2,0.75,1.5)$} & \multicolumn{3}{|c|}{ set $4=(2,2,0.9,1.5)$} \\
\hline \multirow{4}{*}{30} & $a$ & 2.0729 & 0.0729 & 0.1626 & 2.0599 & 0.0599 & 0.1557 \\
\hline & $\alpha$ & 2.1398 & 0.1398 & 0.4224 & 2.1500 & 0.1500 & 0.3931 \\
\hline & $\beta$ & 0.8028 & 0.0528 & 0.0506 & 0.9570 & 0.0570 & 0.0681 \\
\hline & $\lambda$ & 1.6183 & 0.1183 & 0.2426 & 1.6936 & 0.1936 & 0.4927 \\
\hline \multirow{4}{*}{50} & $a$ & 2.0409 & 0.0409 & 0.0910 & 2.0381 & 0.0381 & 0.0882 \\
\hline & $\alpha$ & 2.0828 & 0.0828 & 0.2004 & 2.0816 & 0.0816 & 0.1914 \\
\hline & $\beta$ & 0.7789 & 0.0289 & 0.0250 & 0.9336 & 0.0336 & 0.0357 \\
\hline & $\lambda$ & 1.5713 & 0.0713 & 0.1257 & 1.6026 & 0.1026 & 0.2039 \\
\hline \multirow{4}{*}{75} & $a$ & 2.0257 & 0.0257 & 0.0569 & 2.0312 & 0.0312 & 0.0595 \\
\hline & $\alpha$ & 2.0511 & 0.0511 & 0.1159 & 2.0478 & 0.0478 & 0.1170 \\
\hline & $\beta$ & 0.7681 & 0.0181 & 0.0156 & 0.9243 & 0.0243 & 0.0238 \\
\hline & $\lambda$ & 1.5447 & 0.0447 & 0.0753 & 1.5613 & 0.0613 & 0.1175 \\
\hline \multirow{4}{*}{100} & $a$ & 2.0214 & 0.0214 & 0.0420 & 2.0187 & 0.0187 & 0.0414 \\
\hline & $\alpha$ & 2.0340 & 0.0340 & 0.0835 & 2.0413 & 0.0413 & 0.0839 \\
\hline & $\beta$ & 0.7636 & 0.0136 & 0.0112 & 0.9171 & 0.0171 & 0.0162 \\
\hline & $\lambda$ & 1.5304 & 0.0304 & 0.0548 & 1.5508 & 0.0508 & 0.0826 \\
\hline
\end{tabular}

The values in Tables 2 and 3 show that, in general, the MSE for the estimates of the parameters $\hat{a}, \hat{\alpha}, \hat{\beta}$ and $\hat{\lambda}$ decreases as $n$ increases. 


\section{TYPE-II CENSORING ESTIMATION}

Consider $X_{(1)}, \ldots, X_{(n)}$ be sample of size $n$ whose life time's have the EWR distribution are set on a life test and the test is stopped at certain number of failure $r$ before all $n$ items have failed. The log likelihood function, denoted by $\ln \ell_{1}$, of TII censoring sample is,

$$
\begin{aligned}
\ln \ell_{1}= & n \ln \frac{n !}{(n-r) !}+n \ln a+n \ln \alpha+n \ln \beta+n \ln \lambda+n \ln 2+\sum_{i=1}^{r} \ln \left(x_{i}\right) \\
& +(\beta-1) \sum_{i=1}^{r} \ln \left(\mathrm{e}^{\lambda x_{i}^{2}}-1\right)+\lambda \sum_{i=1}^{r} x_{i}^{2}-\alpha \sum_{i=1}^{r}\left(\mathrm{e}^{\lambda x_{i}^{2}}-1\right)^{\beta} \\
& +(a-1) \sum_{i=1}^{r} \ln \left[1-\exp \left[-\alpha\left(\mathrm{e}^{\lambda x_{i}^{2}}-1\right)^{\beta}\right]\right]+(n-r) \ln \left(1-\left[1-\exp \left[-\alpha\left(\mathrm{e}^{\lambda x_{r}^{2}}-1\right)^{\beta}\right]\right]^{a}\right) .
\end{aligned}
$$

Note that; for simplicity we write $x_{i}$ instead of $x_{(i)}$. The ML estimators of $a, \alpha, \beta$ and $\lambda$ based on TII censored sample are obtained by solving the non-linear equations which we get it from first derivatives of $\ln \ell_{1}$, with respect to parameters $a, \alpha, \beta$ and $\lambda$.

Table 4. Parameter estimator of EWR distribution under TII censored samples

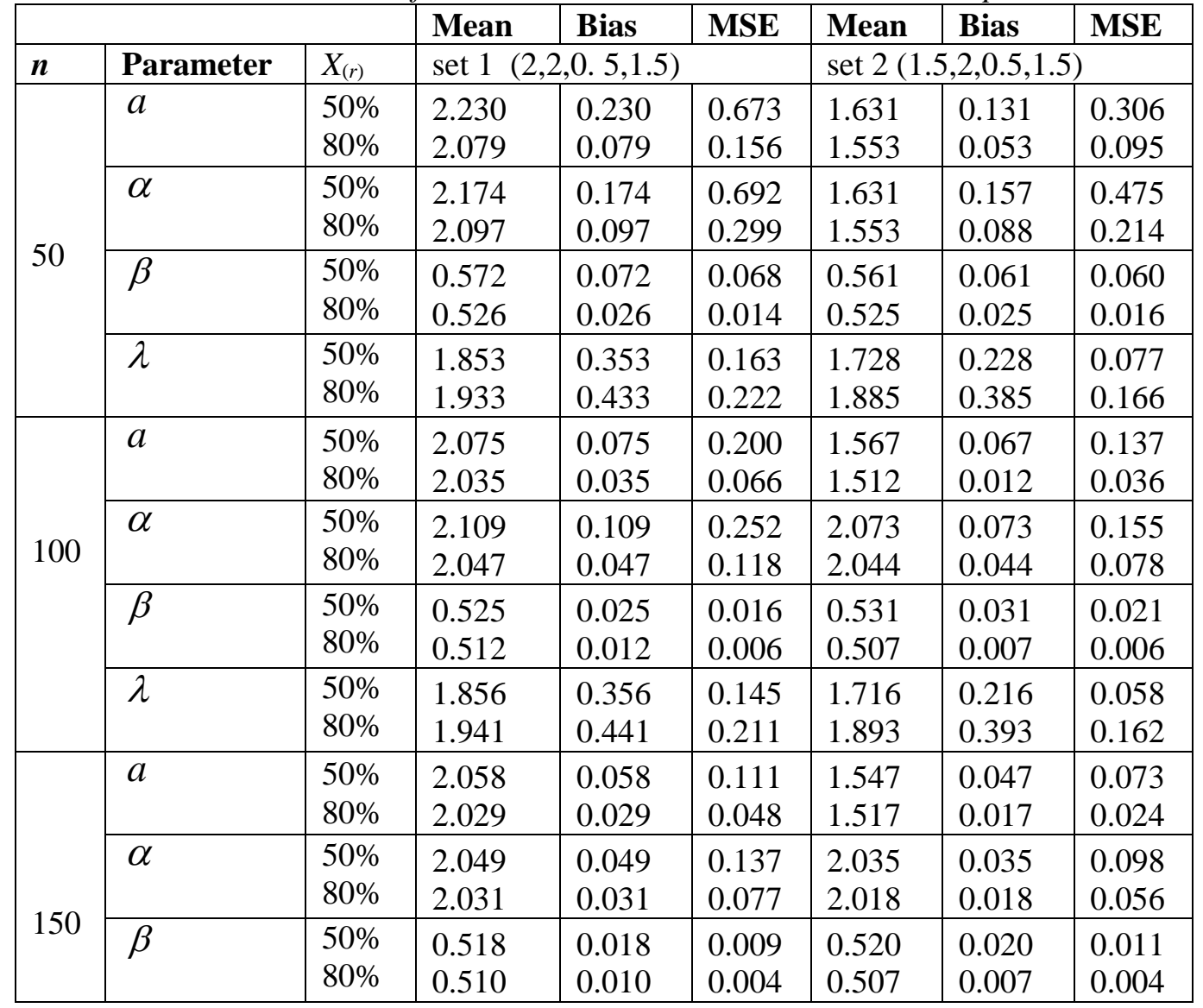




\begin{tabular}{|l|l|l|l|l|l|l|l|l|}
\hline & $\lambda$ & $50 \%$ & 1.845 & 0.345 & 0.130 & 1.709 & 0.209 & 0.051 \\
& & $80 \%$ & 1.944 & 0.444 & 0.209 & 1.889 & 0.389 & 0.157 \\
\hline
\end{tabular}

From Table 4, we see that as $n$ increases the MSE tend to decrease.

\section{APPLICATIONS}

Two real data sets are utilized to show that the EWR model outperforms some of other-models. These models are the EW Weibull (EWW) that introduced in [14], WR, Rayleigh Rayleigh (RR) and exponential Rayleigh (ER) dsitributions.

We use formal goodness-of-fit criteria for the purpose of comparison. These criteria are as the minus value of $\ln \ell$, multiplied by 2, Kolmogorov-Smirnov (K-S) test and its $p$-value, Akaike information criterion (AIC), the corrected AIC (CAIC), the Bayesian information criterion (BIC) and the Hannan-Quinn information criterion (HQIC). The better fitness corresponds to largest $p$-value and the smallest values for all other meaures.

\section{Example 1.}

The first data represents 30 successive values of March precipitation (in inches) for Minneapolis/St Paul (see [23]). Table 5 provides the MLEs of parameters of the EWR, EWW, WR, RR and ER distributions and their standard errors (S.Es). While, the goodness-of-fit measures are mentioned in Table 6.

Table 5. The MLEs and the S.Es (in parentheses) of the model parameters for the first data set

\begin{tabular}{|c|c|c|c|c|c|}
\hline \multirow[b]{2}{*}{ Model } & \multicolumn{4}{|c|}{ MLEs and SEs } & \multirow[b]{2}{*}{$\hat{\gamma}$} \\
\hline & $\hat{a}$ & $\hat{\alpha}$ & $\hat{\beta}$ & $\hat{\lambda}$ & \\
\hline EWR & $\begin{array}{l}3.322 \\
(0.02659)\end{array}$ & $\begin{array}{l}4.918 \\
(0.164)\end{array}$ & $\begin{array}{l}0.468 \\
(0.086)\end{array}$ & $\begin{array}{l}0.042 \\
(0.00629)\end{array}$ & \\
\hline EWW & $\begin{array}{l}78.61 \\
(0.14836)\end{array}$ & $\begin{array}{l}79.35 \\
(0.561)\end{array}$ & $\begin{array}{l}20.486 \\
(0.131)\end{array}$ & $\begin{array}{l}0.624 \\
(0.024)\end{array}$ & $\begin{array}{l}0.014 \\
(0.148)\end{array}$ \\
\hline WR & & $\begin{array}{l}3.888 \\
(0.08635)\end{array}$ & $\begin{array}{l}0.792 \\
(0.015)\end{array}$ & $\begin{array}{l}0.043 \\
(0.02)\end{array}$ & \\
\hline $\mathbf{R R}$ & & $\begin{array}{l}100.351 \\
(0.21297)\end{array}$ & & $\begin{array}{l}0.014 \\
(0.00229)\end{array}$ & \\
\hline ER & & $\begin{array}{l}9.151 \\
(0.09666)\end{array}$ & & $\begin{array}{l}0.025 \\
(0.00306)\end{array}$ & \\
\hline
\end{tabular}

We notice that the EWR distribution yields a better fit than other-models. It has largest $p$-value and the smallest values for the other measures. Plots of the fitted densities and cdfs of the considered distributions are represented in Figure 5. As seen from this figure, that the EWR distribution outperforms the WR, RR and ER distributions.

Table 6. The goodness-of-fit statistics for the first data set

\begin{tabular}{|l|l|l|l|l|l|r|r|}
\hline Distribution & $-2 \ln \ell$ & AIC & CAIC & BIC & HQIC & \multicolumn{1}{|l|}{ K-S } & $\boldsymbol{p}$-value \\
\hline EWR & 114.703 & 122.703 & 124.303 & 122.132 & 126.329 & 0.062 & 0.99985 \\
\hline EWW & 129.022 & 139.022 & 141.522 & 136.407 & 141.263 & 0.113 & 0.83514 \\
\hline WR & 127.973 & 133.973 & 134.896 & 132.405 & 135.318 & 0.088 & 0.97545 \\
\hline RR & 243.627 & 247.627 & 248.071 & 247.341 & 249.439 & 0.426 & 0.00004 \\
\hline ER & 139.679 & 143.679 & 144.124 & 142.634 & 144.576 & 0.12 & 0.78339 \\
\hline
\end{tabular}



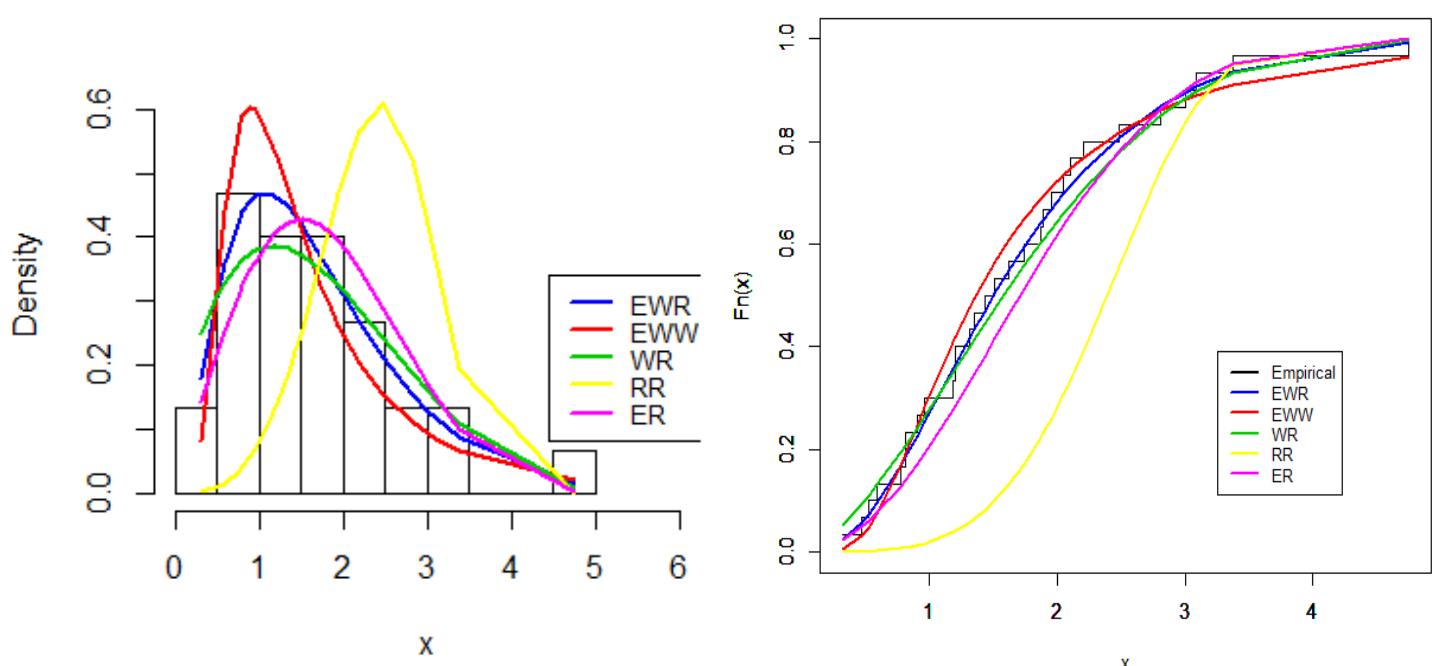

Figure 5. The fitted cdfs (left) and pdfs (right) of the EWR, WR, RR and ER distributions of first data

\section{Example 2.}

We consider the second data which represent the survival times (in days) of 72 guinea pigs infected with different amout of virulent tubercle bacilli (see [24]). Table 7 contains the MLEs of the parameters of the considered models and their corresponding S.Es. While Table 8 displays the goodness-of-fit statistics.

Table 7. The MLEs and the S.Es (in parentheses) of the parameters for the second data set

\begin{tabular}{|c|c|c|c|c|c|}
\hline \multirow[b]{2}{*}{ Model } & \multicolumn{4}{|c|}{ MLEs } & \\
\hline & $\hat{a}$ & $\hat{\alpha}$ & $\hat{\beta}$ & $\hat{\lambda}$ & $\hat{\gamma}$ \\
\hline EWR & $\begin{array}{l}1.415 \\
(0.0078) \\
\end{array}$ & $\begin{array}{l}3.17 \\
(0.105) \\
\end{array}$ & $\begin{array}{l}0.648 \\
(0.057) \\
\end{array}$ & $\begin{array}{l}0.051 \\
(0.00405) \\
\end{array}$ & \\
\hline EWW & $\begin{array}{l}115.001 \\
(0.0787) \\
\end{array}$ & $\begin{array}{l}125.918 \\
(0.361) \\
\end{array}$ & $\begin{array}{l}19.125 \\
(0.085) \\
\end{array}$ & $\begin{array}{l}0.61 \\
(0.015)\end{array}$ & $\begin{array}{l}0.013 \\
(0.093) \\
\end{array}$ \\
\hline WR & - & $\begin{array}{l}4.9 \\
(0.05586)\end{array}$ & $\begin{array}{l}0.809 \\
(0.015) \\
\end{array}$ & $\begin{array}{l}0.031 \\
(0.011)\end{array}$ & \\
\hline RR & - & $\begin{array}{l}25.417 \\
(0.13753) \\
\end{array}$ & - & $\begin{array}{l}0.022 \\
(0.00147)\end{array}$ & \\
\hline ER & & $\begin{array}{l}12.812 \\
(0.06241)\end{array}$ & & $\begin{array}{l}0.016 \\
(0.00197)\end{array}$ & \\
\hline
\end{tabular}

Table 8. The goodness-of-fit statistics for the second data set

\begin{tabular}{|l|r|r|r|r|r|r|r|}
\hline Distribution & \multicolumn{1}{|c|}{$\ln \ell$} & \multicolumn{1}{|c|}{ AIC } & CAIC & BIC & HQIC & \multicolumn{1}{|c|}{ K-S } & \multicolumn{1}{c|}{-value } \\
\hline EWR & 257.833 & 265.833 & 266.43 & 265.263 & 269.459 & 0.097 & 0.50777 \\
\hline $\boldsymbol{E W W}$ & 302.076 & 312.076 & 312.972 & 311.363 & 316.608 & 0.134 & 0.14963 \\
\hline WR & 265.356 & 271.356 & 271.709 & 270.928 & 274.075 & 0.117 & 0.27833 \\
\hline RR & 552.88 & 556.88 & 557.054 & 556.595 & 558.693 & 0.506 & 0.0000 \\
\hline ER & 321.478 & 325.478 & 325.652 & 325.193 & 327.291 & 0.146 & 0.09292 \\
\hline
\end{tabular}

We observe the EWR distribution gives the best fit among the other selected models. The fitted pdfs and cdfs plots of mentioned distributions for the second data are represented in Figure 6 for the purpose visual comparison. According to these plots, we observe that the EWR distribution is prefable than other distributions. 

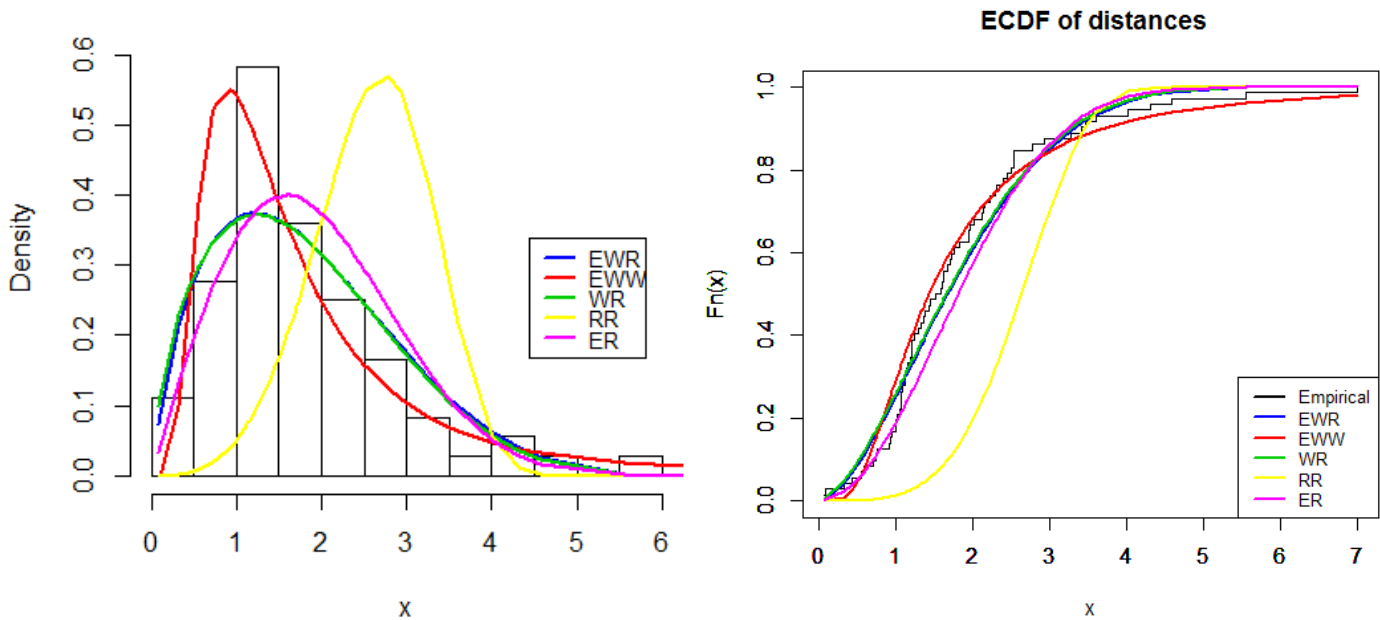

Figure 6. The fitted cdfs (left) and pdfs (right) of the EWR, WR, RR and ER distributions for the second data set

\section{CONCLUSION}

A four-parameter exponentiated Weibull Rayleigh distribution is introduced. Some certain properties of the proposed distribution are discussed. This model includes some new special distributions. The practical importance of the EWR model is clarified through two applications, where the EWR distribution yields the best fit among the else regarded models. We conclude that the EWR distribution can be regarded as a quite flexible gadget for modelling a big group of life data. We trust that the new distribution may serve as rather model to else life distributions and has application in lots of scientific fields.

\section{APPENDIX A}

Theorem 1. Suppose that $(\Omega, F, \boldsymbol{P})$ is a given probability space and let $H=[a, b]$ is an interval for some $d<$ $b\left(a=-\infty, b=\infty\right.$ might as well be allowed): Let X $: \Omega \rightarrow \mathrm{H}$ be a continuous r.v. with cdf F. Also, suppose $q_{1}$ and $q_{2}$ are two real functions defined on $H$, where

$$
E\left[q_{2}(X) \mid X \geq x\right]=E\left[q_{1}(X) \mid X \geq x\right] \eta(x), \quad x \in H,
$$

is defined with some real function $\eta$. Further, let $q_{1}, q_{2} \in C^{1}(\mathrm{H}), \eta \in C^{2}(\mathrm{H})$, and $F$ is twice continuously differentiable and strictly monotone function on the set $H$. Finally, assume that the equation $\eta q_{1}=q_{2}$ has no real solution in the interior of $H$. Then $F$ is uniquely determined by the functions $q_{1}, q_{2}$ and $\eta$, specifically,

$$
F(x)=\int_{a}^{x} C\left|\frac{\eta^{\prime}(u)}{\eta(u) q_{1}(u)-q_{2}(u)}\right| \mathrm{e}^{-s(u)} d u,
$$

where the function is a solution of differential equation $s^{\prime}(x)=\frac{\eta^{\prime} q_{1}}{\eta q_{1}-q_{2}}$. and $C$ is the normalization constant, such that $\int_{H} d F=1$.

\section{CONFLICTS OF INTEREST}

No conflict of interest was declared by the authors. 


\section{REFERENCES}

[1] Eugene, N., Lee, C., Famoye, F., "Beta-normal distribution and its applications", Communication in Statistics-Theory and Methods, 31: 497-512,(2002).

[2] Cordeiro, G.M., de Castro, M., "A new family of generalized distributions", Journal of Statistical Computation and Simulation, 81: 883-893, (2011).

[3] Bourguignon, M., Silva, R.B., Cordeiro, G.M., "The Weibull-G family of probability distributions", Journal of Data Science, 12: 53-68, (2014).

[4] Elgarhy, M., Hassan, A.S., Rashed, M., "Garhy- generated family of distributions with application", Mathematical Theory and Modeling, 6:1-15, (2016).

[5] Hassan, A.S., Elgarhy, M., "A new family of exponentiated Weibull-generated distributions", International Journal of Mathematics and its Applications, 4: 135-148, (2016b).

[6] Hassan, A.S., Hemeda, S.E., "The additive Weibull-g family of probability distributions", International Journals of Mathematics and its Applications, 4: 151-164, (2016).

[7] Hassan, A.S., Elgarhy., M., "Kumaraswamy Weibull-generated family of distributions with applications", Advances and Applications in Statistics, 48: 205-239, (2016a).

[8] Hassan, A.S., Elgarhy, M., Shakil, M., "Type II half logistic family of distributions with applications", Pakistan Journal of Statistics and Operation Research, 13(2): 245-264, (2017).

[9] Elgarhy., M., Haq, M.A., Ozel, G., Nasir, M.A., "A new exponentiated extended family of distributions with applications", Gazi University Journal of Science, 30: 101-115, (2017).

[10] Kong, L., Lee, C., Sepanski, J.H., "On the properties of beta-gamma distribution”, Journal of Modern Applied Statistical Methods, 6: 187-211, (2007).

[11] Merovci, F., Elbatal, I., "Weibull Rayleigh distribution: theory and applications", Applied Mathematics and Information Sciences, 5: 1-11, (2015).

[12] MirMostafaee, S.M.T.K., Mahdizadeh, M., Lemonte, A.J., "The Marshall-Olkin extended generalized Rayleigh distribution: Properties and applications", Communications in Statistics-Theory and Methods, 46: 653-671, (2017).

[13] Kenney, J. F., Keeping, E., “Mathematics of Statistics. D”, Van Nostr and Company, (1962).

[14] Moors, J. J. A., “A quantile alternative for kurtosis”, Journal of the Royal Statistical Society, Series D, (The Statistician), 37(1): 25-32, (1988).

[15] Kotz, S., Shanbhag, D.N., "Some new approaches to probability distributions", Advances in Applied Probability, 12: 903-921, (1980).

[16] Navarro, J., Franco, M., Ruiz, J.M., "Characterization through moments of the residual life and conditional spacing", Sankhya: The Indian Journal of Statistics, Series A, 60: 36-48, (1998).

[17] Kundu, C., Nanda, A.K.,"Some reliability properties of the inactivity time", Communications in Statistics-Theory and Methods, 39: 899-911, (2010).

[18] Zenga, M., "Inequality curve and inequality index based on the ratios between lower and upper arithmetic means", Statistica e Applicazioni 4: 3-27, (2007). 
[19] David, H.A., Nagaraja, H.N., "Order statistics", 3rd edition, John Wiley \& Sons, Hoboken, New Jersey,(2003).

[20] Glänzel, W., "A characterization theorem based on truncated moments and its application to some distribution families", Mathematical Statistics and Probability Theory (Bad Tatzmannsdorf, 1986), Vol. B, Reidel, Dordrecht, 75-84, (1987).

[21] Glänzel, W., "Some consequences of a characterization theorem based on truncated moments", Statistics: A Journal of Theoretical and Applied Statistics, 21: 613-618, (1990).

[22] Hamedani, G.G., "On certain generalized gamma convolution distributions II”, Technical Report, No. 484, MSCS, Marquette University, (2013).

[23] Hinkley, D., "On quick choice of power transformations", Journal of the Royal Statistical Society, Series (c), Applied Statistics, 26:67-69, (1977).

[24] Bjerkedal, T., "Acquisition of resistance in guinea pigs infected with different doses of virulent tubercle bacilli”, American Journal of Epidemiology, 72(1): 130-48, (1960). 